\title{
Training effect of exchange bias in $\gamma-\mathrm{Fe}_{2} \mathrm{O}_{3}$ coated $\mathrm{Fe}$ nanoparticles
}

\author{
R. K. Zheng, G. H. Wen, K. K. Fung, and X. X. Zhang* \\ Department of Physics and Institute of Nano Science and Technology, The Hong Kong University of Science and Technology, \\ Clear Water Bay, Kowloon, Hong Kong, China
}

(Received 11 January 2004; revised manuscript received 13 April 2004; published 28 June 2004)

\begin{abstract}
Training effect of exchange bias in the $\gamma-\mathrm{Fe}_{2} \mathrm{O}_{3}$ coated $\mathrm{Fe}$ nanoparticles were studied. The experimental results indicate that the frozen spins in $\gamma-\mathrm{Fe}_{2} \mathrm{O}_{3}$ shells are responsible for both the horizontal and vertical shifts of the field-cooling hysteresis loops. To understand exchange bias and training effect in the nanoparticles, we modified the Stoner-Wohlfarth model by adding the unidirectional anisotropy energy term to the total energy. It is found that the exchange bias and training effect in the nanoparticles can be well interpreted within the modified model. Since the configuration of the frozen spins was gradually varying as the applied field cycled for the hysteresis loop measurements, the number of the spins frozen along the cooling-field direction decreased, consequently, the exchange bias field $H_{E}$ became smaller.
\end{abstract}

DOI: 10.1103/PhysRevB.69.214431

PACS number(s): 75.60.- d, 75.75.+a, 75.50.Lk

\section{INTRODUCTION}

A magnetic system containing the ferromagnetic/ antiferromagnetic (FM/AFM) interface may exhibit exchange bias effect ${ }^{1,2}$ characterized by the horizontal shift of the hysteresis loops after the system is cooled down through the critical temperature in an external field. Exchange bias effect has been intensively studied both theoretically and experimentally due to its extensive applications in magnetic sensors and data storage. ${ }^{3,4}$ However, up to now, there has not been a theoretical model ${ }^{1,5}$ that can account for all the diverse experimental results. ${ }^{1,2}$

One of the interesting characteristics of exchange bias is that it shows a strong dependence on the thermal and magnetic histories. ${ }^{6}$ The initial temperature and the applied magnetic field in the field-cooling procedure essentially influence exchange bias. ${ }^{7}$ The consecutive hysteresis loop measurements after field cooling show that the exchange bias fields $H_{E}$ decrease with ordinal measurement cycles, known as the training effect. ${ }^{8,9}$ On one side, the understanding of the training effect requires the exact mechanism of exchange bias. On the other side, the study of the training effect can really help to understand exchange bias.

In this paper, we report the study on exchange bias in the $\gamma-\mathrm{Fe}_{2} \mathrm{O}_{3}$ coated $\mathrm{Fe}$ nanoparticles (NP's), in which the $\gamma$ $-\mathrm{Fe}_{2} \mathrm{O}_{3}$ shells show a spin-glass-like behavior. The amount of the aligned frozen spins in $\gamma-\mathrm{Fe}_{2} \mathrm{O}_{3}$ plays a crucial role in exchange bias. Exchange bias and the training effect in the nanoparticles are well explained in the framework of the modified Stoner-Wohlfarth model. ${ }^{10}$

\section{EXPERIMENTS}

Fe NP's in this study were prepared using a plasma-gascondensation-type deposition system. ${ }^{11} \mathrm{~A}$ dc power of $240 \mathrm{~W}$ was applied to a sputtering gun with a high purity iron foil as the target to create the plasma in a vacuum chamber filled with $\mathrm{Ar}$ gas of 0.66 Torr. In the high pressure $\mathrm{Ar}$ gas, the sputtered Fe atoms nucleate and form small clusters. In order to obtain particles with a narrow size distribution, the clusters were extracted by the pressure difference through a series of collimated apertures and collected in another chamber with a pressure of $10^{-4}$ Torr. After the deposition, the sample chamber was slowly filled with air to passivate the particles. The chemical state of surface oxide of the nanoparticles was studied by $\mathrm{x}$-ray photoelectron spectroscopy (XPS) with the monochromatic Al $K \alpha$ x-ray source after energy calibration referring to the $\mathrm{C} 1 \mathrm{~s}$ peak. High resolution transmission electron microscopy (HRTEM) observations and electron diffraction analysis of the nanoparticles were carried out using a JEOL 2010F analytical TEM operated at $200 \mathrm{kV}$. Magnetic measurements were made by a Quantum Design SQUID with RSO option in the field from $-50-50 \mathrm{kOe}$ over the temperature from 2 to $350 \mathrm{~K}$.

\section{RESULTS AND DISCUSSIONS}

The high-resolution XPS spectrum of the particles was obtained in order to determine valance of the $\mathrm{Fe}$ ion in the oxide shell, as shown in Fig. 1(a). The satellite peak at $719 \mathrm{eV}$ is clearly seen in the spectrum of the $\mathrm{Fe} 2 p$ region, which reveals that the surface oxide is $\mathrm{Fe}_{2} \mathrm{O}_{3}$. Shown in Figs. 1(b) and 1(c) are the TEM image of the particles and the corresponding electron diffraction patterns. It is seen that the particles are quite uniform, or with a relatively narrow size distribution. A close inspection of the electron diffraction pattern reveals that it is composed of two sets of diffraction rings. One set is identified to be from $\alpha$-Fe and the other face-centered-cubic style is from surface oxide. This, combined with the XPS results, allows us to conclude that the surface oxide is $\gamma-\mathrm{Fe}_{2} \mathrm{O}_{3}$. The detailed core/shell structure, $5 \mathrm{~nm} \mathrm{Fe}$ core covered by the $3 \mathrm{~nm}$ oxide shell, and the lattice image of core and shell are shown in the HRTEM image, Fig. 1(d).

To explore the intrinsic properties of the particles, the low field $(0.01 \mathrm{~T})$ zero-field cooled (ZFC) and field cooled (FC) magnetization curves were measured as the following. The sample was first cooled in zero-field from 300 to $5 \mathrm{~K}$, then a $0.01 \mathrm{~T}$ field was applied, after that the magnetic moment was recorded with temperature increasing from 5 to $300 \mathrm{~K}$, the 


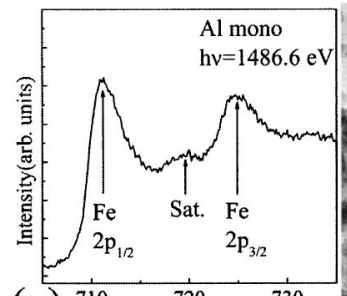

(a) ${ }^{710}{ }_{\text {Binding Energy (ev) }}^{730}$
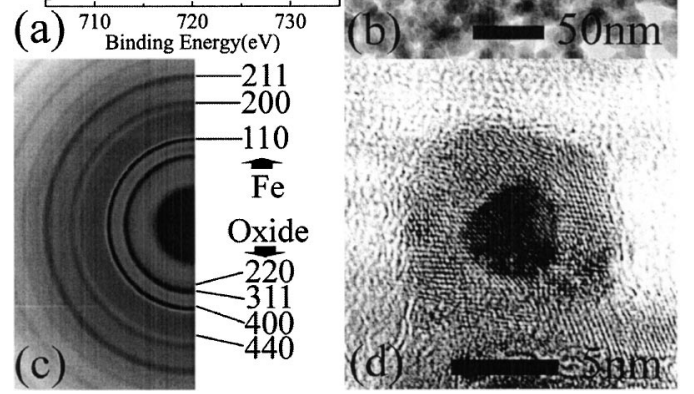

FIG. 1. (a) The Fe $2 p$ region of the high-resolution XPS spectrum of particles. (b) The TEM image showing the narrow size distribution of the particles. (c) The corresponding electron diffraction pattern, which is composed of two sets of diffraction rings. (d) The HRTEM image of a typical particle, a $5 \mathrm{~nm} \alpha$-Fe core covered by a $3 \mathrm{~nm} \gamma-\mathrm{Fe}_{2} \mathrm{O}_{3}$ shell.

ZFC curve. The magnetic moment was measured again by cooling the sample to $5 \mathrm{~K}$ and keeping the applied field unchanged. The resulted curve is FC (cooling). The FC (warming) is the magnetic moment measured by heating the sample again to $300 \mathrm{~K}$ in the same field. The ZFC, FC (cooling), and FC (heating) curves are shown in Fig. 2. The ZFC curve shows a broad peak at around $110 \mathrm{~K}$, which indicated a broad energy distribution. ${ }^{12}$ The peak temperature is normally called the blocking temperature $T_{B}(=110 \mathrm{~K})$. The FC (cooling) curve is nearly superposed on the one of FC (heating), which indicates that the FC curve, measured in the slow temperature sweep rate of $1.0 \mathrm{~K} / \mathrm{min}$, represents the equilibrium states of the system.

It has been recognized that the surface layers of ferrites may enter the spin-glass-like state at low temperature. ${ }^{8,13,14}$ To observe this phenomenon in the $\mathrm{Fe} / \gamma-\mathrm{Fe}_{2} \mathrm{O}_{3}$ nanoparticles, the ZFC, FC (cooling), and FC (heating) curves were measured with a high field of $5 \mathrm{~T}$ and shown in the inset of

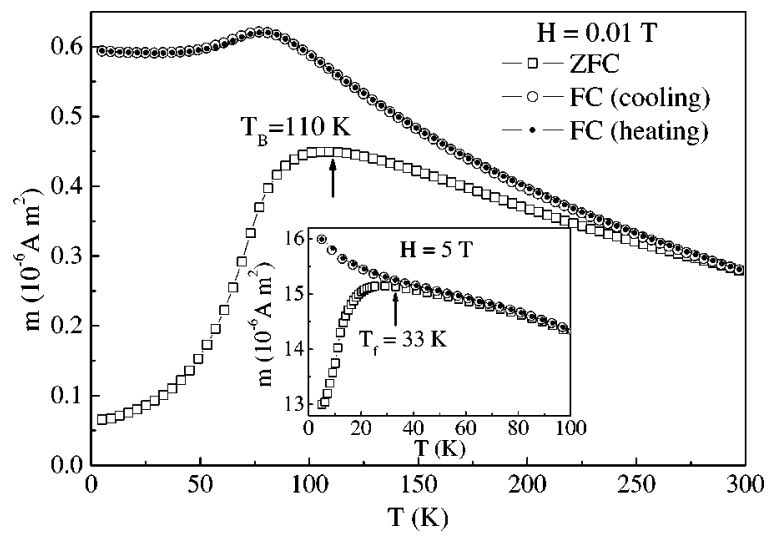

FIG. 2. The ZFC-FC curves measured in a $0.01 \mathrm{~T}$ field. Inset: The ZFC-FC curves measured in a $5 \mathrm{~T}$ field.

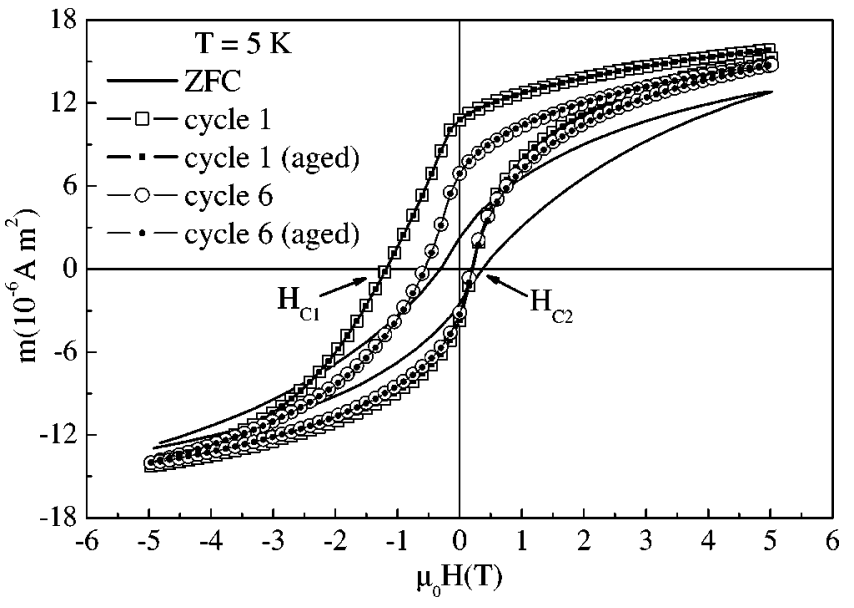

FIG. 3. The ZFC loop, first and sixth FC loops, and aged first and sixth FC loops at $5 \mathrm{~K}$. All the loops show the irreversibility up to $5 \mathrm{~T}$.

Fig. 2. The FC (cooling) and FC (heating) curves are again undistinguishable. The divergence of the ZFC and FC curves below $33 \mathrm{~K}$ indicates that part of the total moment is frozen and cannot be reversed even in a field as large as $5 \mathrm{~T}$. Since the anisotropy fields of the $\mathrm{Fe}$ and $\gamma-\mathrm{Fe}_{2} \mathrm{O}_{3}$ are all much smaller than $5 \mathrm{~T}^{8}{ }^{8}$ the high field divergence may be resulted from the spin-glass-like phase as reported prevously, $, 13,14$ whose freezing temperature $T_{f}$ is at or above $33 \mathrm{~K}$. The spinglass-like phase can exist at the surfaces of $\gamma-\mathrm{Fe}_{2} \mathrm{O}_{3}$, interfaces between $\mathrm{Fe}$ and $\gamma-\mathrm{Fe}_{2} \mathrm{O}_{3}, \gamma-\mathrm{Fe}_{2} \mathrm{O}_{3}$ grain boundaries, and the regions with impurities or structural disorders. In canonical spin glass, the irreversibility is usually suppressed in a field as high as $5 \mathrm{~T} .{ }^{15,16}$ However, in the spin-glass-like phase of $\mathrm{NiFe}_{2} \mathrm{O}_{4}$, the irreversibility existed even in a $16 \mathrm{~T}$ field due to the surface spin disorder and surface anisotropy. ${ }^{13,14,17}$ It will be shown below that this high-field irreversibility play a crucial role in the exchange bias of the nanoparticles.

The ZFC magnetic hysteresis loop was measured after the nanoparticles were cooled in zero-field from $T=300 \mathrm{~K}$ [above $T_{B}$ and $T_{f}$ but below the Curie temperatures $T_{C}$ $=1043 \mathrm{~K}$ of $\mathrm{Fe}$ (Ref. 18) and $T_{C}=900 \mathrm{~K}$ of $\gamma-\mathrm{Fe}_{2} \mathrm{O}_{3}$ (Ref. 19)] to $5 \mathrm{~K}$. To study the training effect of exchange bias, the nanoparticles were first cooled in a field of $5 \mathrm{~T}$ from $T$ $=300$ to $5 \mathrm{~K}$, and then six complete hysteresis loops were recorded. The ZFC and first and sixth FC loops are shown in Fig. 3. All the loops are open even in a field up to $5 \mathrm{~T}$, which is known as high field irreversibility. It is also seen from the loops that the magnetic moment is not saturated even in fields as high as $5 \mathrm{~T}$, which is indicative of the existence of the spin-glass-like phase. ${ }^{14,20}$ Most importantly both the exchange bias field $H_{E}$ and the magnetic moment decrease clearly with magnetic field cycling. Namely, the training effect is obviously observed in the nanoparticles. To clarify the time effect on hysteresis loops, we adopted the FC loop measurements method except that an aging of $10^{4} \mathrm{~s}$ is inserted before each hysteresis loop measurement. The first and sixth aged FC loops were also shown in the Fig. 3. The aged FC loops are almost the same as those without aging, which indicate that time effect is neglectable in comparison with that of the field training. 


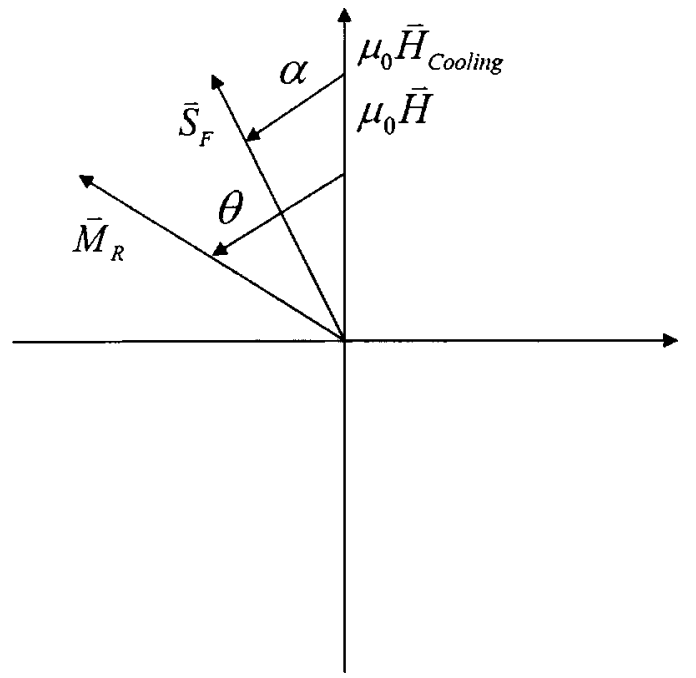

FIG. 4. The modified Stoner-Wohlfarth model and the defini-

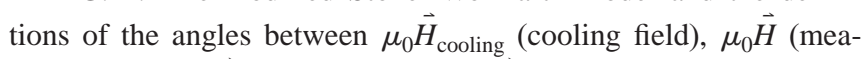
surement field), $\vec{S}_{F}$ (frozen spin), and $\vec{M}_{R}$ (reversible magnetization or reversible spin $\vec{S}_{R}$ ).

In order to display exchange bias, a system must contain, at least, two exchange coupled phases: one reversible phase that can be reversed and one fixed phase that cannot be reversed in the field range of measurements. However, in the $\gamma-\mathrm{Fe}_{2} \mathrm{O}_{3}$ coated $\mathrm{Fe}$ nanoparticle system, neither Fe nor $\gamma$ $-\mathrm{Fe}_{2} \mathrm{O}_{3}$ can act as the fixed phase to provide the reversible phase with pinning energy. It is the spin-glass-like phase with high-field irreversibility in $\gamma-\mathrm{Fe}_{2} \mathrm{O}_{3}$ shell that can play the role of the fixed phase in the $\mathrm{Fe} / \gamma-\mathrm{Fe}_{2} \mathrm{O}_{3}$ system. When the system is cooled down below $T_{f}$ in an external field, some of the spins of the spin-glass-like phase are aligned to and frozen in the direction of the cooling field. In the following hysteresis loop measurements, some of the frozen spins keep their directions and provide pinning force to the reversible spins. As a result, exchange bias appears.

Now we consider exchange bias and training effect within one particle. The $\gamma-\mathrm{Fe}_{2} \mathrm{O}_{3}$ coated Fe nanoparticle of $11 \mathrm{~nm}$ in diameter can be treated as a single domain particle. We try to describe the reversal of the magnetization of the nanoparticle by modifying Stoner-Wohlfarth model. ${ }^{10}$ The definitions of vectors and angle are shown in Fig. 4. Let $N_{F}$ and $N_{R}$ denote the numbers of the frozen spins $\vec{S}_{F}$ and reversible spins $\vec{S}_{R}$ per unit volume in one particle, respectively. Since the reversal of magnetization in Stoner-Wohlfarth model is by coherent rotation, all the reversible spins should have the same direction as the reversible magnetization $\vec{S}_{R} \| \vec{M}_{R}$. But the directions of $\vec{S}_{F}$ depend on the thermal and field history. The total energy of the original Stoner-Wohlfarth model has two terms. The first term is the uniaxial anisotropy energy. For the $\mathrm{Fe} / \gamma-\mathrm{Fe}_{2} \mathrm{O}_{3}$ nanoparticles, the anisotropy term $E_{\text {an }}$ should include the contributions of all the anisotropies except the unidirectional anisotropy of exchange bias. The second term is Zeeman energy $-\mu_{0} \vec{H} \cdot \vec{M}_{R}$. For an exchange bias system, the pinning energy that leads to the unidirectional anisotropy $K_{\mathrm{UD}}$ should be added in the total energy. For sim- plicity, we assume the effective exchange couplings supplied by one frozen spin is $J_{\text {eff. }}$ The pinning energy per unit volume is given by

$$
E_{p}=-\sum_{i=1}^{N_{F}} J_{\text {eff }} \cos \left(\alpha_{i}-\theta\right)
$$

So, the total energy per unit volume in an exchange bias particle can be written as

$$
E=E_{\text {an }}-\mu_{0} \vec{H} \cdot \vec{M}_{R}-\sum_{i=1}^{N_{F}} J_{\text {eff }} \cos \left(\alpha_{i}-\theta\right) .
$$

In the situation of $\mathrm{ZFC}$, the directions of the frozen spins in a particle are parallel to the magnetization of the particle, because the exchange interaction between the frozen and reversible spins is actually established due to the fact that $T_{B}$ is higher than $T_{f}$ in this system. Since the easy axes of the particles are randomly distributed in space (random distribution of $\theta$ ), the unidirectional anisotropy resulting from exchange must also be randomly distributed. In this case, the unidirectional anisotropy effect from each particle will be cancelled each other, leading to a symmetrical hysteresis loop as shown in Fig. 3.

In the situation of $\mathrm{FC}$, the directions of the frozen spins are aligned in the field direction, which generate effective pinning energy. Let $N_{F}^{\text {net }}$ denote the number of the net frozen spins per unit volume. The pinning energy can be written as $N_{F}^{\text {net }} J \cos \theta$, or in the form of unidirectional anisotropy ${ }^{21,22}$ $K_{\mathrm{UD}} \cos \theta=N_{F}^{\mathrm{net}} J_{\text {eff }} \cos \theta$. The exchange bias field is determined by

$$
H_{E}=K_{\mathrm{UD}} / \mu_{0} M_{R}=N_{F}^{\mathrm{net}} J_{\mathrm{eff}} / \mu_{0} M_{R}
$$

For the measurement field $\vec{H}$ parallel to the cooling field $\vec{H}_{\text {cooling }}$, the total energy is

$$
E=E_{\mathrm{an}}-\mu_{0}\left(|\vec{H}|+H_{E}\right) M_{R} \cos \theta
$$

For the measurement field $\vec{H}$ antiparallel to the cooling field $\vec{H}_{\text {cooling }}$, the total energy is

$$
E=E_{\text {an }}-\mu_{0}\left(-|\vec{H}|+H_{E}\right) M_{R} \cos \theta .
$$

As well known, the hysteresis loop is determined by minimizing the total energy. The coercivity at the cooling field direction is decreased by $H_{E}$ from Eq. (4); the coercivity at the opposite direction of cooling field is increased by $H_{E}$ from Eq. (5). In other words, the unidirectional anisotropy horizontally shifts the FC loop to the opposite direction of cooling field by $H_{E}$. The amount of the net frozen spins $N_{F}^{\text {net }}$ is proportional to the difference in the measured magnetization at +5 and $-5 \mathrm{~T}$ :

$$
N_{F}^{\mathrm{net}} \propto \Delta M(5 \mathrm{~T})=M(5 \mathrm{~T})+M(-5 \mathrm{~T})
$$

Training effect can be well interpreted within this model. As is well known, one of the characteristics of spin glass is its multiple configuration of the ground state. ${ }^{15}$ Some of the frozen spins, which were originally aligned in the cooling field direction, may change their directions and fall into other 
metastable configurations during the hysteresis measurements. In other words, $N_{F}^{\text {net }}$ decreases with the field cycling. This characteristic of spin-glass-like phase essentially influences the exchange bias behaviors of the system. One of the consequences of the reconfiguration of the aligned frozen spins is the decrease of magnetic moment difference $\Delta M(5 T)$ according to Eq. (6). Another one is the decrease of $H_{E}$ according to Eq. (3).

If the differences of $J_{\text {eff }}$ for different frozen spins are ignored, one can express $H_{E}$ with $\Delta M$ from Eqs. (4)-(6):

$$
H_{E} \propto \Delta M(5 T) / M_{R} .
$$

The reduced magnetization difference at $5 \mathrm{~T}$, $\Delta M(5 \mathrm{~T}) / M_{R}(5 \mathrm{~T})$, were extracted from experimental data of the six hysteresis loops. The $H_{E}$ dependence on $\Delta M(5 T) / M_{R}(5 T)$ is plotted in Fig. 5. The approximately linear dependence manifests the crucial role of the frozen spins in the determination of the exchange bias field.

\section{CONCLUSION}

Exchange bias and training effect is observed in the $\gamma$ $-\mathrm{Fe}_{2} \mathrm{O}_{3}$ coated Fe Nanoparticles. Both the exchange bias and training effect were well explained in the modified StonerWohlfarth model. The field-cooling hysteresis shifts in both the horizontal and vertical directions can be associated with the frozen spins, whose configuration changes with the field

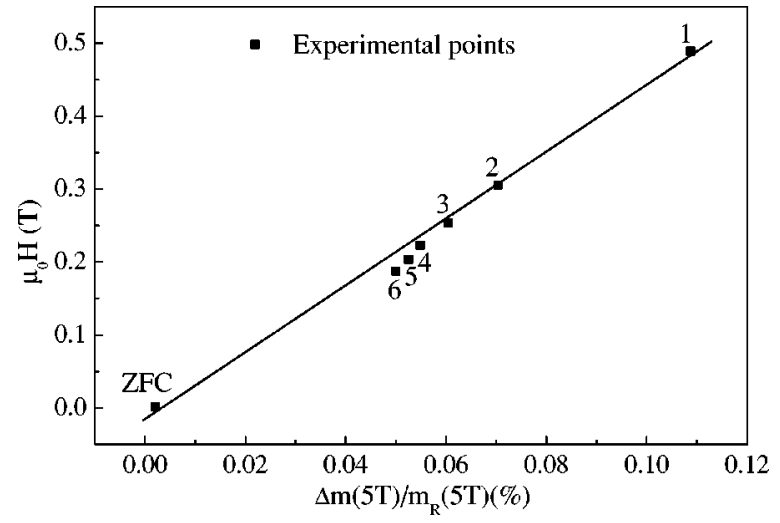

FIG. 5. The dependence of $H_{E}$ on $\Delta M(5 \mathrm{~T}) / M_{R}(5 \mathrm{~T})$. The line is a guide to the eyes.

cycling during the hysteresis loop measurements. The decrease of the frozen spins along the cooling field direction reduces the effective pinning energy. Consequently, $H_{E}$ decreases with the field cycling.

\section{ACKNOWLEDGMENTS}

This work was partially supported by grants from the RGC of the Hong Kong (Project No. HKUST6165/01P, HKUST6140/00P).
*Email address: phxxz@ust.hk

${ }^{1}$ J. Nogues and I. K. Schuller, J. Magn. Magn. Mater. 192, 203 (1999).

${ }^{2}$ A. E. Berkowitz and K. Takano, J. Magn. Magn. Mater. 200, 552 (1999).

${ }^{3}$ J. C. S. Kools, IEEE Trans. Magn. 32, 3165 (1996).

${ }^{4}$ S. S. P. Parkin, K. P. Roche, M. G. Samant, P. M. Rice, R. B. Beyers, R. E. Scheuerlein, E. J. O'Sullivan, S. L. Brown, J. Bucchigano, D. W. Abraham, Y. Lu, M. Rooks, P. L. Trouilloud, R. A. Wanner, and W. J. Gallagher, J. Appl. Phys. 85, 5828 (1999).

${ }^{5}$ M. Kiwi, J. Magn. Magn. Mater. 234, 584 (2001).

${ }^{6}$ N. J. Gokemeijer, J. W. Cai, and C. L. Chien, Phys. Rev. B 60, 3033 (1999).

${ }^{7}$ N. J. Gokemeijer and C. L. Chien, J. Appl. Phys. 85, 5516 (1999).

${ }^{8}$ B. Martinez, X. Obradors, L. Balcells, A. Rouanet, and C. Monty, Phys. Rev. Lett. 80, 181 (1998).

${ }^{9}$ S. G. E. te Velthuis, A. Berger, G. P. Felcher, B. K. Hill, and E. D. Dahlberg, J. Appl. Phys. 87, 5046 (2000).

${ }^{10}$ E . C. Stoner and E. P. Wohlfarth, Philos. Trans. R. Soc. London, Ser. A 240, 599 (1948).
${ }^{11}$ G . H. Wen, R. K. Zheng, K. K. Fung, and X. X. Zhang, J. Magn. Magn. Mater. 270, 407 (2004).

${ }^{12}$ X. X. Zhang, J . M. Hernandez, J. Tejada, and R. F. Ziolo, Phys. Rev. B 54, 4101 (1996).

${ }^{13}$ R. H. Kodama, A. E. Berkowitz, E. J. McNiff, and S. Foner, Phys. Rev. Lett. 77, 394 (1996).

${ }^{14}$ R. H. Kodama, A. E. Berkowitz, E. J. McNiff, and S. Foner, J. Appl. Phys. 81, 5552 (1997).

${ }^{15}$ K. Binder and A. P. Young, Rev. Mod. Phys. 58, 801 (1986).

${ }^{16}$ J. A. Mydosh, Spin Glasses: An Experimental Introduction (Taylor \& Francis, London, 1993).

${ }^{17}$ R. H. Kodama and A. E. Berkowitz, Phys. Rev. B 59, 6321 (1999).

${ }^{18}$ Robert C. O’Handley, Modern Magnetic Materials: Principles and Applications (John Wiley \& Sons, New York, 2000).

${ }^{19}$ Robert C. O'Handley, Modern Magnetic Materials: Principles and Applications (John Wiley \& Sons, New York, 2000).

${ }^{20}$ R. H. Kodama, S. A. Makhlouf, and A. E. Berkowitz, Phys. Rev. Lett. 79, 1393 (1997).

${ }^{21}$ W. H. Meiklejohn and C. P. Bean, Phys. Rev. 102, 1413 (1956).

${ }^{22}$ W. H. Meiklejohn and C. P. Bean, Phys. Rev. 105, 904 (1957). 